\title{
Effect of Unilateral Low-Frequency Stimulation of Hippocampus on Rapid Kindling-Induced Seizure Development in Rats
}

\author{
Lucas Toibaro, Magdalena Pereyra, Julieta Pastorino, Ariela Smigliani, Florencia Ocariz, \\ Germán Ortmann, María Milagros Galardi, María Belén Gori, Silvia Kochen*
}

Epilepsy Laboratory, Institute of Cellular Biology and Neuroscience-National Council for Scientific and Technical Research (CONICET), School of Medicine, University of Buenos Aires, Buenos Aires, Argentina.

Email: *skochen@retina.ar

Received February 10 ${ }^{\text {th }}, 2012$; revised March 13 ${ }^{\text {th }}, 2012$; accepted April $5^{\text {th }}, 2012$

\begin{abstract}
Since the last decade deep brain stimulation has been proposed as an alternative treatment for patients who do not become seizure-free with the current pharmacological treatments and cannot undergo resective surgical procedure. However, the optimal stimulation parameters remain undetermined and active research in humans and animals is necessary. The present study was designed to investigate the effect of unilateral Low Frequency Stimulation (LFS) of hippocampus on seizure development by using the hippocampal rapid kindling method (hRK) in rats. We used male Wistar rats implanted with electrodes in the ventral hippocampus. All rats underwent hRK (biphasic square wave pulses, $20 \mathrm{~Hz}$ for 10 seconds) during three consecutive days (twelve stimulations per day). The control group $(\mathrm{hRK} ; \mathrm{n}=6)$ received only RK stimulus, while the treated group (LFS-hRK; $\mathrm{n}=8$ ) received also LFS (biphasic square wave pulses, $1 \mathrm{~Hz}$ for 30 seconds) immediately before the RK stimulus, during three consecutive days. At the end of behavioral testing on day 3 , $62 \%(\mathrm{P}<0.05)$ of the animals receiving LFS treatment were still not fully kindled staying in stages 0 -III $(\mathrm{P}<0.01)$. The number of stimulations needed to achieve generalized seizures (stage IV-V of Racine scale) was significantly higher ( $\mathrm{P}$ $<0.05$ ) in the LFS group with respect to control group. No significant differences in the cumulative daily afterdischarge duration were observed between both groups. These findings suggest that preemptive LFS can significantly decrease the incidence of hippocampus-kindled seizures and delay the progression and secondary generalization of focal seizures.
\end{abstract}

Keywords: Hippocampal Rapid Kindling; Epilepsy; Electrical Stimulation; Low-Frequency Stimulation; Electrical Stimulation Protective Effect

\section{Introduction}

Since the last decade deep brain stimulation (DBS) has been proposed as an alternative treatment for patients with drug-resistant epilepsy and are not amendable to resective surgery $[1,2]$. DBS of various neural targets has been investigated in clinical studies and animal studies, including the anterior nucleus of thalamus (ANT) [3-6], subthalamic nucleus $[7,8]$, centromedian nucleus of the thalamus $[9,10]$, caudate nucleus [11], cerebellum [1214], and hippocampus [15-18]. Despite this broad range of researches, the optimal stimulation parameters as well as the neuroanatomical target remain uncertain [19]. Therefore, active research in both humans and animals is necessary.

The stimulation of hippocampus may provide an alternative to patients who have refractory temporal lobe

\footnotetext{
${ }^{*}$ Corresponding author.
}

epilepsy (TLE). Moreover, stimulation of hippocampus, as ictal onset zone, could potentially avoid memory loss associated with resective surgery.

Kindling is probably the most widely accepted experimental model for TLE [20]. Traditional kindling protocols usually provide once- or twice-daily stimulations and depending on the region that is stimulated, it takes 10 - 30 days for the rats to become fully kindled. Lothman et al. (1988/93/94) described the serial day rapid kindling (RK) procedure and found that the serial day rapid kindling protocol renders fully kindled animals faster than traditional kindling [21-23].

The inhibitory effect of low frequency stimulation (LFS) has been widely proved using the conventional kindling method, in animal model of complex partial epilepsy $[24,25]$. Several studies have used LFS of the kindling focus [26] as well as neuroanatomical structures related to the genesis and propagation of the ictal activity 
[27,28], proving both of them inhibition of the kindling acquisition and seizure development with controversial results. However, there are few reports of the effect of LFS in hippocampus using the rapid kindling (RK) method. The experimental results published by Mohammad-Zadeh et al. [29] and Wyckhuys et al. [30] showed that the optimal LFS duration remains indeterminate and that there are still arguable results in relation to its efficacy.

Taking into account the reduced time required to develop the kindling process, three days in the protocol that we used [31], the RK is a fast alternative to conventional kindling and could be considered as a more practical experimental model than the conventional kindling method (which requires approximately 30 days to fulfil the experiment).

Considering the important role of hippocampus in epileptogenesis and due to there are no sufficient reports about the effect of LFS on hippocampus in a rapid kindling protocol, the present study was designed to investigate the inhibitory efficacy of unilateral LFS in a model of hippocampal RK (hRK) in wistar rats.

\section{Materials and Methods}

\subsection{Animals}

Adult male Wistar rats $(220-270 \mathrm{~g})$ were housed individually in cages with an ambient temperature of $23^{\circ} \mathrm{C}$ $25^{\circ} \mathrm{C}$ and a 12 -h light/12-h dark cycle (lights on from 6:00 - 18:00 h). Animals were provided with water and food ad libitum. They were acclimated for at least 1 week before surgery. Experiments were carried out each day between 10:00 - 17:00 $\mathrm{h}$.

The experimental protocol was approved by the Institutional Committee for the Care and Use of Laboratory Animals (CICUAL) of School of Medicine of Buenos Aires University and was in complete accordance with the UE (86/609/EEC) and the ethical guidelines of the Committee of Health Guide for the Care and Use of Laboratory Animals of School of Veterinary Medicine of Buenos Aires University. Furthermore, efforts were made to minimize the number of animals used in the study and their suffering.

\subsection{Surgery}

The rats were deeply anaesthetized with intraperitoneal injections of a ketamine/xylazine mixture at a concentration of $85.0 \mathrm{mg} / \mathrm{ml}$ ketamine and $15.0 \mathrm{mg} / \mathrm{ml}$ xylazine. The rats were fixed in a stereotaxic frame according to the method of Paxinos and Watson [32] and the skull was exposed. Five holes were drilled, two of them for anchor screws, two for placement of a stainless steel wire used as ground and reference and one for placement of the electrode. A bipolar recording and stimulating electrode $(0.2 \mathrm{~mm}$ in diameter) was chronically implanted in the right ventral hippocampus, using the following coordinates in $\mathrm{mm}$ from bregma and skull surface: anteroposterior -5.3 ; lateral 5.2; depth 7.5 [32]. The electrodes consisted in two twisted stainless steel Teflon-coated wires (tip distance $0.5-1.0 \mathrm{~mm}$ ) insulated except for $0.5 \mathrm{~mm}$ at the tip. Electrode was connected to a miniature receptacle, which was embedded in the skull with dental cement. At least 7 days were allowed for recovery from surgery before starting the experiment. Following completion of the experimental protocol, all animals were anaesthetized by an intraperitoneal injection of ketamine $(85 \mathrm{mg} / \mathrm{kg})$ and xylazine $(15 \mathrm{mg} / \mathrm{kg})$ and then transcardially perfused with $4 \%$ paraformaldehyde. Brains were frozen and coronally sectioned into $40 \mu \mathrm{m}$ slices with a microtome and slide mounted, allowing for confirmation of electrode placements. The data of the animals with false placement of their electrode position or existence of any abnormality, such as lesion, were not included in the results.

\subsection{Stimulation Procedures}

All the recordings were performed after the rat had been transferred from the home cage to a recording box $(30 \times$ $30 \times 30 \mathrm{~cm}$ ). The head-stage of the rat was connected to a flexible, shielded cable. Evoked responses were recorded in freely moving rat while the animal was awake with its eyes open.

Seven to ten days post surgical recovery, on day 0 , the afterdischarge thresholds (ADT) were determined as the minimum intensity sufficient to evoke an afterdischarge (AD) of $5 \mathrm{~s}$ or longer, defined as a two-fold increase in the EEG baseline. An initial current of $50 \mu \mathrm{A}$ (peak-tobase; $2 \mathrm{~s}$ trains of biphasic square wave pulses, $20 \mathrm{~Hz}$ ) was applied and then increased in steps of $50 \mu \mathrm{A}$ (to a maximum of $400 \mu \mathrm{A}$ ), with 5 minutes intervals between current delivery. The animals that did not elicit AD with a current intensity of $400 \mu \mathrm{A}$ were excluded from the experiment. On day 1 the implanted animals were assigned to two groups: the control group $(\mathrm{hRK} ; \mathrm{n}=6)$ and the treated group with LFS (LFS $+\mathrm{hRK} ; \mathrm{n}=8)$. All animals received the rapid kindling stimulus. The hRK stimulus consisted in 12 stimulations daily during three consecutive days. Each stimulus was carried out at ADT intensity with $10 \mathrm{~s}$ trains of biphasic square wave pulses, at a frequency of $20 \mathrm{~Hz}$ with intervals of 30 minutes between each of them. The LFS (biphasic square wave pulses $50 \mu \mathrm{A}, 1 \mathrm{~Hz}$ for 30 seconds) was applied to the treated group immediately before each of the $12 \mathrm{RK}$ stimulations on three stimulation days. LFS parameters were determined according to Goodman et al. [26] and 
our preliminary experiments (unpublished data, 2010).

Electroencephalogram (EEG) during each $\mathrm{AD}$ was recorded through the hippocampal electrodes. All of the recording were obtained and reviewed by using a digital system (Stellate-Bioscience system, Bioscience S.R.L, Buenos Aires, Argentina). The signal was amplified 1000 times, filtered $0.5-40 \mathrm{~Hz}(3 \mathrm{~dB} /$ octave $)$, and digitized at a sampling rate of $200 \mathrm{~Hz}$. The behavioral manifestation was classified following an adjusted version of the scale of Racine [25]: stage I: immobility, facial clonus, wet dog shakes; stage II: head nodding, chewing, automatisms; stage III: clonus of one forelimb; stage IV: rearing, bilateral forelimb clonus; stage V: rearing, bilateral forelimb clonus, loss of balance and falling [25,33,34]. Stages I-III were considered as focal seizures, while stages IV and V were considered as generalized seizures (GS) [35-37]. When the animals exhibited three consecutive stage 5 seizures, they were regarded as fully kindled. An investigator who had no information about the animal group and the number of stimulations scored the behavioral responses. After the completion of experiment, the incidence of GS, the mean of stimulations necessaries to reach the stage IV and V (generalization of seizures), the behavioral progression of kindling (stages I-V; according to Racine's scores), and the cumulative daily AD (ADD) were monitored to evaluate the effect of the LFS during the kindling acquisition. Values for ADD were calculated by summing the duration of ADs recorded after each of the daily twelve stimulations.

\subsection{Statistical Analysis}

A two-way analysis of variance (ANOVA) followed by a post hoc Bonferroni's test was done to compare the ADD and the number of stimulation required to achieve different stages between hRK (control) and LFS + hRK groups. The behavioral progression of kindling (stages $\mathrm{I}-\mathrm{V}$ ) was analyzed by using Mann Whitney U test. In the case of comparing generalized seizure incidence, chisquare test was used. Data are expressed as mean and standard error of the mean (SEM). Statistical analysis was carried out by GraphPad Prism 4.0 for Windows. For all analysis, the tests were performed two-sided and a $\mathrm{P}<$ 0.05 was considered significant.

\section{Results}

Fourteen of fifteen rats used in this experiment fulfilled the criteria, 6 were assigned to the control group (hRK) and 8 to the treated group (LFS $+\mathrm{hRK}$ ). At the end of experiment, all control rats were fully kindled, while in LFS group only 3 of 8 rats (38\%) arrived generalized seizures (stage IV-V) but they did not become fully kindled ( $\mathrm{P}<0.05$; Chi-square test) (Figure 1(a)). Five ani- mals of LFS (62\%) were still not fully kindled staying in stages 0 -III being observed a significant difference on day 3 ( $\mathrm{P}<0.01$; Mann Whitney $\mathrm{U}$ test) with respect to control group (Figure 1(b)).

A two-way ANOVA and post hoc Bonferroni's test showed that the average of stimulation required to reach stage IV-V (generalized seizures) was significantly higher in LFS + hRK group $(26.17 \pm 3.90)$ than in control group $(17.75 \pm 0.95)(\mathrm{P}<0.05$; Figure 2$)$. However, post hoc Bonferroni's test did not show a significant effect of LFS on the ADD, although LFS group showed a decreasing ADD from first to last day, but did not reach significance (Figure 3).

\section{Discussion}

The obtained data show that LFS application with the parameters used in the current study interferes with hRK.

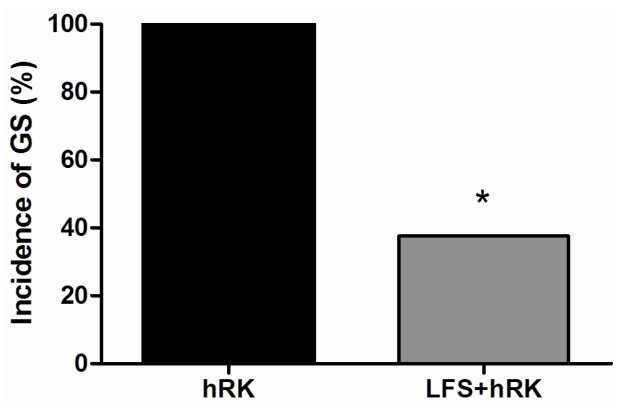

(a)

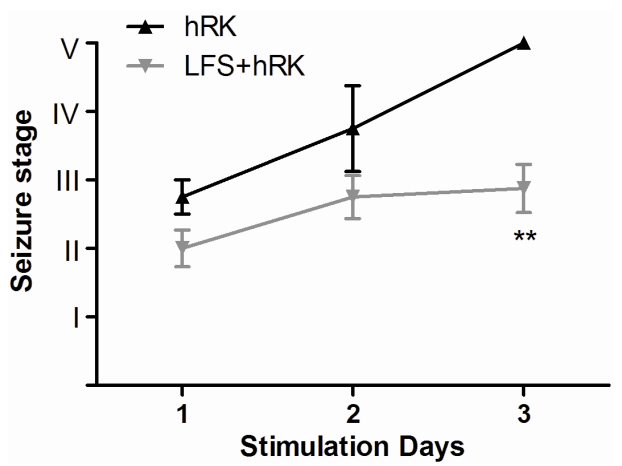

(b)

Figure 1. Effect of LFS on seizure severity. (a) hRK group (control; black bar) and LFS treated group (gray bar) on the incidence of generalized seizures in hippocampal-kindled rats. Chi-Square test showed that LFS-treated group (LFS + hRK) had significantly less incidence of generalized seizures (GS) than control group (hRK; ${ }^{*} \mathrm{P}<\mathbf{0 . 0 5}$ ), reaching GS only the $38 \%$ of LFS + hRK animals; (b) Effect of LFS application on progression of behavioral seizure stages during 3 days of stimulations in treated group (LFS + hRK) compared to control group (hRK). The nonparametric MannWhitney $U$ test showed a significant difference on behavioral seizure scores on day $3\left(^{* *} \mathrm{P}<0.01\right)$. Data were shown as mean \pm SEM. 


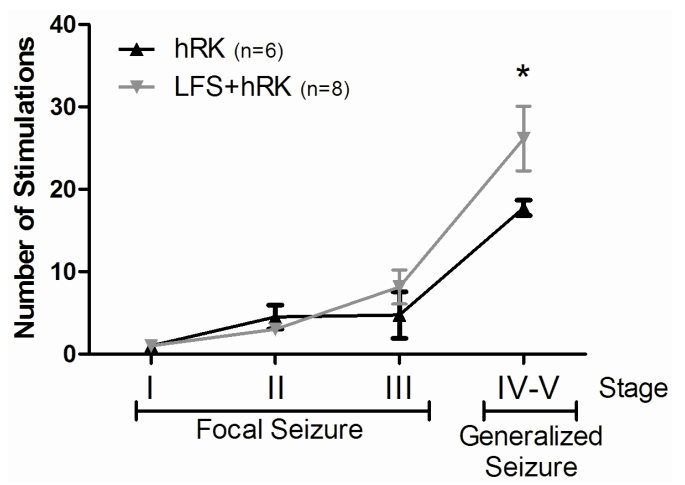

Figure 2. Effect of LFS application on number of stimulations needed to achieve different seizure stages. A two-way ANOVA followed by a post hoc Bonferroni's test showed that the average number of stimulations required to reach generalized seizures (stages IV and V) were significantly increased in LFS + hRK group compared with control group (hRK; *P < 0.05). Data were shown as mean \pm SEM ( $n=6$ for hRK group and $n=8$ for LFS + hRK group).

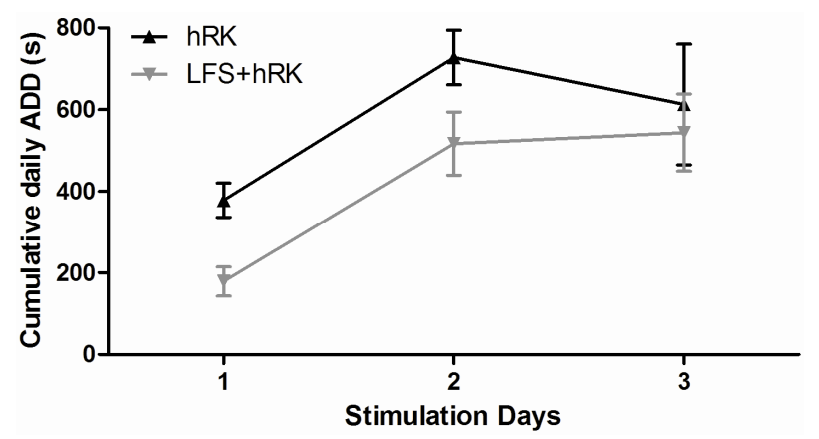

Figure 3. Effect of LFS application on progression of $\mathrm{Cu}-$ mulative daily afterdischarge duration (daily ADD). The table shows the cumulative daily ADD of control (hRK) and treated (LFS + hRK) groups. A two-way ANOVA, followed by a post hoc Bonferroni's test, did not show significant differences between both groups. These data are plotted as cumulative daily ADD (seconds) versus days of stimulation. Data were shown as mean \pm SEM.

The LFS group presented a significant difference to reached stages of generalized seizures (IV and V) in relation to control group. Therefore, at the end of behavioral testing, $62 \%$ of the animals receiving LFS treatment were still not fully kindled remaining in stages 0-III. LFS significantly slowed the progression of behavioral seizure stage on day 3 showing a retardation of kindling acquisition.

The LFS significantly increase the number of stimulations required to achieve generalized seizures. This effect is predominantly due to the significant retardation of progression from stages 0-III (focal seizures) to stages IV and $\mathrm{V}$ (generalized seizures). However, ADD was not affected by LFS.

The LFS applied in RK model on perforant path [29,
38] and in amygdala-kindled rats [26] showed an anticonvulsant effect similar to our findings. Other researchers did not found anticonvulsant effect of LFS on seizure parameters using RK in piriform cortex [39]. On the other hand, no anticonvulsant effect of LFS in hRK was observed by Wyckhuys et al. [30], but they used a different protocol, the alternate day RK and a higher frequency of stimulation $(5 \mathrm{~Hz}$ instead of $1 \mathrm{~Hz}$ used in our work). This controversy about the LFS antiepileptic efficacy could be due to the different parameters used in each experiment. It is known that different parameters of LFS have important role in induction of LFS anticonvulsant effects. It seems that the slower LFS frequency and the shorter interval between LFS and kindling stimulations could cause the stronger anticonvulsant effect [40]. Therefore, this effect depends on the applied LFS parameters (e.g. intensity, pulse duration and train duration) and the designed protocol [39].

Recently published results from the SANTE (Stimulation of the Anterior Nuclei of Thalamus for Epilepsy) trial demonstrate a significant reduction in seizure frequency with programmed stimulation $[4,12,41,42]$ making the anterior nuclei of thalamus (ANT) a promising target in seizure control for intractable epilepsy $[6,43,44]$. However, more large and well-controlled studies should seek to apply stimulation to patients with inefficacious effect and those were excluded in SANTE.

The hippocampus is another promising target for intractable epilepsy. There is important evidence to consider hippocampus as one of the most epilepsy-susceptible areas and the origin of temporal lobe epilepsy [43]. However there are few reports about this area as stimulation target $[18,22,30]$, we applied LFS in this structure, assuming that the inhibition of seizure onset site would facilitates the blocking of epileptic discharge.

In the present report, it has been demonstrated that using the hRK method can be observed an anticonvulsive effect of LFS during kindling acquisition showing antiepileptogenic potential. It has not been yet determined the mechanisms that contribute to the strong protective effect of LFS but it has been hypothesized that electrical stimulation of specific brain areas can regulate seizure susceptibility by raising seizure threshold or by interfering with seizure propagation by activating seizure-gating networks [44-46]. A few studies using LFS in the kindling focus have indicated that LFS-induced LTD or depotentiation may reverse kindling-induced long-term potentiation in the focus, so retarding seizure development [47,48]. The LFS induced-decrease in kindled seizures severity observed in the present study can be explained by an increase in $\mathrm{AD}$ threshold $[26,46]$. An elevation in seizure threshold would make it less likely that a given kindling stimulus would elicit a seizure. Therefore, if 
kindling occurs through a potentiation of excitatory pathways, then an LFS-induced increase in seizure threshold may occur through a depotentiation-like process possibly delaying network synchronization and blocking seizure generalization. This also could explain why the treated animals needed more stimulations than controls to achieve fully kindled state.

However, ADD is decreased during application of LFS, but failed to reach significance. As in our report, it has been previously observed by Wyckhuys et al. (2010) that neither HFS nor LFS could affect the ADD despite having increased the $\mathrm{AD}$ latency and $\mathrm{AD}$ threshold. Other reports of rapid kindling applied during more of three consecutive days of stimulation $[29,30,38,40]$ showed LFS-induced decreased ADD which differ of our findings. Therefore, no significant change in ADD between treated and control animals may be due to that time of protocol used in this study (three days) do not allow observed a long-lasting effect of LFS. Further experiments using a prolonged protocol of rapid kindling will be performed.

\section{Conclusion}

The hRK could be considered as a more practical experimental model than the conventional kindling method. Preemptive LFS can gives significant retardation of progression from stages III (focal seizures) to stages IV-V (generalized seizures). These findings may afford a new insight into establishing an effective and long-lasting therapy for seizure prone people, especially those with temporal lobe epilepsy in future.

\section{Acknowledgements}

This study was funded by UBACYT, School of Medicine, University of Buenos Aires.

\section{REFERENCES}

[1] B. C. Jobst, "Brain Stimulation for Surgical Epilepsy," Epilepsy Research, Vol. 89, No. 1, 2010, pp. 154-161. doi:10.1016/j.eplepsyres.2009.08.017

[2] A. Schulze-Bonhage, "Deep Brain Stimulation: A New Approach to the Treatment of Epilepsy," Deutsches Ärzteblatt International, Vol. 106, No. 24, 2009, pp. 407-412. doi:10.3238/arztebl.2009.0407

[3] M. A. Mirski, L. A. Rossell, J. B. Terryb and R. S. Fisherc, "Anticonvulsant Effect of Anterior Thalamic High Frequency Electrical Stimulation in the Rat," Epilepsy Research, Vol. 28, No. 2, 1997, pp. 89-100. doi:10.1016/S0920-1211(97)00034-X

[4] M. Hodaie, R. A. Wennberg, J. O. Dostrovsky and A. M. Lozano, "Chronic Anterior Thalamus Stimulation for Intractable Epilepsy," Epilepsia, Vol. 43, No. 6, 2002, pp.

\section{3-608. doi:10.1046/j.1528-1157.2002.26001.x}

[5] I. Osorio, J. Overman, J. Giftakis and S. B. Wilkinson, "High Frequency Thalamic Stimulation for Inoperable Mesial Temporal Epilepsy," Epilepsia, Vol. 48, No. 8, 2007, pp. 1561-1571. doi:10.1111/j.1528-1167.2007.01044.X

[6] X. L. Zhong, K. R. Lv, Q. Zhang, J. T. Yua, Y. Y. Xing, N. D. Wangd and L. Tan, "Low-Frequency Stimulation of Bilateral Anterior Nucleus of Thalamus Inhibits Amygdale-Kindled Seizures in Rats," Brain Research Bulletin, Vol. 86, No. 5-6, 2011, pp. 422-427. doi:10.1016/j.brainresbull.2011.08.014

[7] N. Usui, S. Maesawa, Y. Kajita, O. Endo, S. Takebayashi and J. Yoshida, "Suppression of Secondary Generalization of Limbic Seizures by Stimulation of Subthalamic Nucleus in Rats," Journal of Neurosurgery, Vol. 102, No. 6, 2005, pp. 1122-1129. doi:10.3171/jns.2005.102.6.1122

[8] A. Handforth, A. A. DeSalles and S. E. Krahl, "Deep Brain Stimulation of the Subthalamic Nucleus as Adjunct Treatment for Refractory Epilepsy," Epilepsia, Vol. 47, No. 7, 2006, pp. 1239-1241. doi:10.1111/j.1528-1167.2006.00563.x

[9] R. S. Fisher, S. Uematsu, G. L. Krauss, B. J. Cysyk, R. McPherson, R. P. Lesser, B. Gordon, P. Schwerdt and M. Rise, "Placebo-Controlled Pilot Study of Centromedian Thalamic Stimulation in Treatment of Intractable Seizures," Epilepsia, Vol. 33, No. 5, 1992, pp. 841-851. doi:10.1111/j.1528-1157.1992.tb02192.x

[10] D. M. Andrade, D. Zumsteg, C. Hamani, M. Hodaie, S. Sarkissian, A. M. Lozano and R. A. Wennberg, "LongTerm Follow-Up of Patients with Thalamic Deep Brain Stimulation for Epilepsy," Neurology, Vol. 66, No. 10, 2006, pp. 1571-1573. doi:10.1212/01.wnl.0000206364.19772.39

[11] S. A. Chkhenkeli, M. Sramka, G. S. Lortkipanidze, T. N. Rakviashvili, E. S. Bregvadze, G. E. Magalashvili, T. S. Gagoshidze and I. S. Chkhenkeli, "Electrophysiological Effects and Clinical Results of Direct Brain Stimulation for Intractable Epilepsy," Clinical Neurology and Neurosurgery, Vol. 106, No.4, 2004, pp. 318-329. doi:10.1016/j.clineuro.2004.01.009

[12] I. S. Cooper, I. Amin, M. Riklan, J. M. Waltz and T. P. Poon, "Chronic Cerebellar Stimulation in Epilepsy. Clinical and Anatomical Studies," Archives of Neurology, Vol. 33, No. 8, 1976, pp. 559-570.

[13] C. Rubio, V. Custodio, F. Juárez and C. Paz, "Stimulation of the Superior Cerebellar Peduncle during the Development of Amygdaloid Kindling in Rats," Brain Research, Vol. 1010, No. 1-2, 2004, pp. 151-155. doi:10.1016/j.brainres.2004.03.015

[14] F. Velasco, J. D. Carrillo-Ruiz, F. Brito, M. Velasco, A. L. Velasco, I. Marquez and R. Davis, "Double-Blind, Randomized Controlled Pilot Study of Bilateral Cerebellar Stimulation for Treatment of Intractable Motor Seizures," Epilepsia, Vol. 46, No. 7,2005, pp. 1071-1081. doi:10.1111/j.1528-1167.2005.70504.x

[15] M. Velasco, F. Velasco and A. L. Velasco, "Centromedian-Thalamic and Hippocampal Electrical Stimulation 
for the Control of Intractable Epileptic Seizures," Journal of Clinical Neurophysiology, Vol. 18, No. 6, 2001, pp. 495-513. doi:10.1097/00004691-200111000-00001

[16] J. F. Tellez-Zenteno, R. S. McLachlan, A. Parrent, C. S. Kubu and S. Wiebe, "Hippocampal Electrical Stimulation in Mesial Temporal Lobe Epilepsy," Neurology, Vol. 66, No. 10, 2006, pp. 1490-1494. doi:10.1212/01.wnl.0000209300.49308.8f

[17] P. Boon, K. Vonck, V. De Herdt, A. Van Dycke, M. Goethals, L. Goossens, M. Van Zandijcke, T. De Smedt, I. Dewaele, R. Achten, W. Wadman, F. Dewaele, J. Caemaert and D. Van Roost, "Deep Brain Stimulation in Patients with Refractory Temporal Lobe Epilepsy," Epilepsia, Vol. 48, No. 8, 2007, pp. 1551-1560. doi:10.1111/j.1528-1167.2007.01005.x

[18] T. Wyckhuys, T. De Smedt, P. Claeys, R. Raedt, L. Waterschoot, K. Vonck, C. Van den Broecke, C. Mabilde, L. Leybaert, W. Wadman and P. Boon, "High Frequency Deep Brain Stimulation in the Hippocampus Modifies Seizure Characteristics in Kindled Rats," Epilepsia, Vol. 48, No. 8, 2007, pp. 1543-1550. doi:10.1111/j.1528-1167.2007.01038.x

[19] B. C. Jobst, T. M. Darcey, V. M. Thadani and D. W. Roberts, "Brain Stimulation for the Treatment of Epilepsy," Epilepsia, Vol. 51, No. S3, 2010, pp. 88-92. doi:10.1111/j.1528-1167.2010.02618.x

[20] G. V. Goddard, "The Kindling Model of Epilepsy," Trends in Neurosciences, Vol. 6, 1983, pp. 275-279. doi:10.1016/0166-2236(83)90118-2

[21] E. W. Lothman, R. A. Salerno, J. B. Perlin and D. L. Kaiser, "Screening and Characterization of Antiepileptic Drugs with Rapidly Recurring Hippocampal Seizures in Rats," Epilepsy Research, Vol. 2, No. 6, 1988, pp. 367379. doi:10.1016/0920-1211(88)90048-4

[22] E. W. Lothman and J. M. Williamson, "Rapid Kindling with Recurrent Hippocampal Seizures," Epilepsy Research, Vol. 14, No. 3, 1993, pp. 209-220. doi:10.1016/0920-1211(93)90045-9

[23] E. W. Lothman and J. M. Williamson, "Closely Spaced Recurrent Hippocampal Seizures Elicit Two Types of Heightened Epileptogenesis: A Rapidly Developing, Transient Kindling and a Slowly Developing, Enduring Kindling," Brain Research, Vol. 649, No. 1-2, 1994, pp. 7184. doi:10.1016/0006-8993(94)91050-2

[24] E. Bertram, "The Relevance of Kindling for Human Epilepsy," Epilepsia, Vol. 48, No. S2, 2007, pp. 65-74.

[25] D. C. McIntyre, M. O. Poulter and K. Gilby, "Kindling: Some Old and Some New," Epilepsy Research, Vol. 50, No. 1-2, 2002, pp. 79-92. doi:10.1016/S0920-1211(02)00071-2

[26] J. H. Goodman, R. E. Berger and T. K Tcheng, "Preemptive Low-Frequency Stimulation Decreases the Incidence of Amygdala-Kindled Seizures," Epilepsia, Vol. 46, No. 1, 2005, pp. 1-7. doi:10.1111/j.0013-9580.2005.03804.x

[27] M. Kinoshita, A. Ikeda, M. Matsuhashi, R. Matsumoto, T. Hitomi T. Begum, K. Usui, M. Takayama, N. Mikuni, S. Miyamoto, N. Hashimoto and H. Shibasaki, "Electric Cortical Stimulation Suppresses Epileptic and Background
Activities in Neocortical Epilepsy and Mesial Temporal Lobe Epilepsy," Clinical Neurophysiology, Vol. 116, No. 6, 2005, pp. 1291-1299.

doi:10.1016/j.clinph.2005.02.010

[28] L. X. Yang, C. L. Jin, Z. B. Zhu-Ge, S. Wang, E. Q. Wei, I. C. Bruce and Z. Chen, "Unilateral Low-Frequency Stimulation of Central Piriform Cortex Delays Seizure Development Induced by Amygdaloid Kindling in Rats," Neuroscience, Vol. 138, No. 4, 2006, pp. 1089-1096. doi:10.1016/i.neuroscience.2005.12.006

[29] M. Mohammad-Zadeh, J. Mirnajafi-Zadeh, Y. Fathollahi, M. Javan, P. Ghorbani, M. Sadegh and S. M. Noorbakhsh, "Effect of Low Frequency Stimulation of Perforant Path on Kindling Rate and Synaptic Transmission in the Dentate Gyrus during Kindling Acquisition in Rats," Epilepsy Research, Vol. 75, No. 2, 2007, pp. 154-161. doi:10.1016/j.eplepsyres.2007.05.003

[30] T. Wyckhuys, R. Raedt, K. Vonck, W. Wadman and P. Boon, "Comparison of Hippocampal DBS with High (130 $\mathrm{Hz}$ ) and Low $(5 \mathrm{~Hz})$ Frequency on Afterdischarges in Kindled Rats," Epilepsy Research, Vol. 88, No. 2-3, 2010, pp. 239-246. doi:10.1016/j.eplepsyres.2009.11.014

[31] T. De Smedt, S. De Rouck, R. Raedt, T. Wyckhuys, L. Waterschoot, V. De Herdt, A. Van Dycke, R. E. Tahry, K. Vonck and P. Boon, "Serial Day Rapid Kindling Is an Effective Tool in Screening the Anti-Epileptic Properties of Topiramate," Seizure, Vol. 16, No. 7, 2007, pp. 620-626. doi:10.1016/j.seizure.2007.04.013

[32] G. Paxinos and C. Watson, "The Rat Brain in Stereotaxic Coordinates," 4th Editon, Academic Press, San Diego, 1998.

[33] R. J. Racine, "Modification of Seizure Activity by Electrical Stimulation: I. Afterdischarge Threshold and II. Motor Seizure," Electroencephalography and Clinical Neurophysiology, Vol. 32, No. 3, 1972, pp. 269-294. doi:10.1016/0013-4694(72)90176-9

[34] M. Sato, R. J. Racine and D. C. McIntyre, "Kindling: Basic Mechanisms and Clinic Validity," Electroencephalography and Clinical Neurophysiology, Vol. 76, No. 5, 1990, pp. 459-472. doi:10.1016/0013-4694(90)90099-6

[35] I. Kanter-Schlifke, B. Georgievska, D. Kirik and M. Kokaia, "Seizure Suppression by GDNF Gene Therapy in Animal Models of Epilepsy," Molecular Therapy: The Journal of the American society of Gene therapy, Vol. 15, No. 6, 2007, pp. 1106-1113.

[36] S. L. Moshé, P. K. Stanton and E. F. Sperber, "Sensitivity of the Immature Central Nervous System to Epileptogenic Stimuli,” In: P. A. Schwartzkroin, Ed., Epilepsy: Models, Mechanisms and Concepts, Cambridge University Press, Cambridge, 1993, p. 173.

[37] S. H. Zhang, H. L. Sun, Q. Fang, K. Zhong, D. C. Wu, S. Wang and Z. Chen, "Low-Frequency Stimulation of the Hippocampal CA3 Subfield Is Anti-Epileptogenic and Anti-Ictogenic in Rat Amygdaloid Kindling Model of Epilepsy," Neuroscience Letters, Vol. 455, No. 1, 2009, pp. 51-55. doi:10.1016/j.neulet.2009.03.041

[38] M. Sadegh, J. Mirnajafi-Zadeh and V. Sheibani, "Serine/ Threonine Protein Phosphatases Have No Role in the In- 
hibitory Effects of Low-Frequency Stimulation in Perforant Path Kindling Acquisition in Rats," Neuroscience Letters, Vol. 451, No. 3, 2009, pp. 266-269. doi:10.1016/j.neulet.2009.01.001

[39] P. Ghorbani, M. Mohammad-Zadeh, J. Mirnajafi-Zadeh and Y. Fathollahi, "Effect of Different Patterns of LowFrequency Stimulation on Piriform Cortex Kindled Seizures," Neuroscience Letters, Vol. 425, No. 3, 2007, pp. 162-166. doi:10.1016/j.neulet.2007.08.023

[40] M. Shahpari, J. Mirnajafi-Zadeh, S. M. P. Firoozabadi and A. Yadollahpour, "Effect of Low-Frequency Electrical Stimulation Parameters on Its Anticonvulsant Action during Rapid Perforant Path Kindling in Rat," Epilepsy Research, Vol. 99, No. 1-2, 2011, pp. 69-77.

[41] I. S. Cooper, A. R. Upton and I. Amin, "Reversibility of Chronic Neurologic Deficits. Some Effects of Electrical Stimulation of the Thalamus and Internal Capsule in Man," Applied Neurophysiology, Vol. 43, No. 3-5, 1980, pp. 244-258.

[42] A. R. Upton, I. Amin, S. Garnett, M. Springman, C. Nahmias and I. S. Cooper, "Evoked Metabolic Responses in the Limbic-Striate System Produced by Stimulation of Anterior Thalamic Nucleus in Man," Pacing and Clinical Electrophysiology, Vol. 10, No. 1, 1987, pp. 217-225. doi:10.1111/j.1540-8159.1987.tb05952.x

[43] X. L. Zhong, J. T. Yu, Q. Zhang, N. D. Wang and L. Tan, "Deep Brain Stimulation for Epilepsy in Clinical Practice and in Animal Models," Brain Research Bulletin, Vol. 85,
No. 3-4, 2011, pp. 81-88.

doi:10.1016/j.brainresbull.2011.03.020

[44] Q. Zhang, Z. C. Wu, J. T. Yu, X. L. Zhong, Y. Y. Xing, Y. T. D. Miao and L. Tan, "Anticonvulsant Effect of Unilateral Anterior Thalamic High Frequency Electrical Stimulation on Amygdala-Kindled Seizures in Rat," Brain Research Bulletin, Vol. 87, No. 2-3, 2012, pp. 221-226. doi:10.1016/j.brainresbull.2011.11.023

[45] K. Gale, "Subcortical Structures and Pathways Involved in Convulsive Seizure Generation," Journal of Clinical Neurophysiology, Vol. 9, No. 2, 1992, pp. 264-277. doi:10.1097/00004691-199204010-00007

[46] D. C. McIntyre, K. Gilby and C. A. Carrington, "Effect of Low-Frequency Stimulation on Amygdala-Kindled Afterdischarge Thresholds and Seizure Profile in Fast and Slow Kindling Rat Strains," Epilepsia, Vol. 48, No. 8, 2002, pp. 1604-1613.

[47] L. Velísek, J. Velísková and P. K. Stanton, "Low-Frequency Stimulation of the Kindling Focus Delays Basolateralamygdala Kindling Inimmature Rats," Neuroscience Letters, Vol. 326, No. 1, 2002, pp. 61-63. doi:10.1016/S0304-3940(02)00294-X

[48] S. R. Weiss, A. Eidsath, X. L. Li, T. Heynen and R. M. Post, "Quenching revisited: Low Level Direct Current Inhibits Amygdala-Kindled Seizures," Experimental Neurology, Vol. 154, No. 1, 1998, pp. 185-192. doi:10.1006/exnr.1998.6932. 